\title{
QUASI-SPHERICAL, TIME-DEPENDENT VISCOUS ACCRETION FLOW: ONE-DIMENSIONAL RESULTS
}

\author{
SeOng-Jae LeE ${ }^{1}$, Dongsu Ryu ${ }^{2}$, and IndRanil Chattopadhyay ${ }^{3}$ \\ ${ }^{1}$ School of Science Education, Chungbuk National University, Chungbuk 361-763, Republic of Korea; seong @ chungbuk.ac.kr \\ ${ }^{2}$ Department of Astronomy and Space Science, Chungnam National University, Republic of Korea \\ ${ }^{3}$ ARIES, Manora Peak, Nainital-263 129, Uttarakhand, India \\ Received 2010 September 28; accepted 2010 December 15; published 2011 February 1
}

\begin{abstract}
We investigated the instability of advective accretion flow as a consequence of angular momentum transfer in one-dimensional, quasi-spherical transonic accretion flow around a non-rotating black hole. The code is designed to include the effects of viscosity; the hydrodynamics component preserves angular momentum strictly with Lagrangian and remap method in the absence of viscosity, while the viscosity component updates viscous angular momentum transfer through the implicit method. We performed two tests to demonstrate the suitability of the code for accretion study. First, we simulated the inviscid, low angular momentum, transonic accretion flow with shocks around a black hole, and then the subsonic, self-similar ADAF solution around a Newtonian object. Both simulations fitted the corresponding analytical curves extremely well. We then simulated a rotating, viscous, transonic fluid with shocks. We showed that for low viscosity parameter, stable shocks at larger distance are possible. For higher viscosity parameter, more efficient angular momentum transfer in the post-shock disk makes the shock structure oscillatory. Moreover, as the shock drifts to larger distances, a secondary inner shock develops. We showed that the inner shock is the direct consequence of the expansion of the outer shock, as well as the creation of regions with $\partial l / \partial r<0$ due to more efficient angular momentum transfer near the inner sonic point. We showed that all disk parameters, including emissivity, oscillate with the same period as that of the shock oscillation. Our simulation may have implications for low frequency quasi-periodic oscillations, e.g., GRO J1655-40 and XTE J1550-564.
\end{abstract}

Key words: accretion, accretion disks - hydrodynamics - instabilities - methods: numerical

\section{INTRODUCTION}

Investigation of the flow behavior of the accreting matter in the vicinity of a black hole is important since the spectrum and intensity of the emitted radiation depend on the flow structure. The event horizon presents the unique inner boundary condition in which the infalling matter crosses the horizon with the speed of light $(c)$. Therefore, black hole accretion has to be transonic, as a result of which the existence of one sonic point or critical point is assured for black hole accretion. General relativity also ensures that matter must possess sub-Keplerian angular momentum closer to the horizon. Although within the marginally stable circular orbit $\left(r_{\mathrm{ms}}\right)$, the angular momentum at $r \lesssim r_{\mathrm{ms}}$ is definitely sub-Keplerian (and the value $l \sim l_{\mathrm{ms}}=\left.l\right|_{r=r_{\mathrm{ms}}}$ ), at larger radius the angular momentum should generally be large. Therefore, a general accretion disk should have viscosity to remove the angular momentum outward. The first serious model of a viscous accretion disk was presented by Shakura \& Sunyaev (1973), in which the angular momentum distribution was Keplerian, and the accretion disk was geometrically thin and optically thick. In the Shakura-Sunyaev disk, the pressure and advection terms were not properly considered and no attempt was made to satisfy the inner boundary condition around a black hole apart from the ad hoc termination of the disk at $r \leqslant r_{\mathrm{ms}}$. Along with this theoretical shortcoming, the Shakura-Sunyaev disk also failed to explain the power-law high energy part of a black hole candidate spectrum. Therefore, the search for another component of an accretion disk, which may explain the origin of the high energy radiations from black hole candidates, was undertaken by various groups. One such model which got a wide attention was ADAF (e.g., Ichimaru 1977; Narayan \& Yi 1994, hereafter NY94). This model was first constructed around a Newtonian gravitational potential, where the viscously dissipated energy is advected along with the mass, momentum, and the entropy of the flow. The original ADAF solution was self-similar and wholly subsonic, and was found to be thermally and dynamically stable. However, the low viscosity ADAF showed convective instability (Igumenshchev \& Abramowicz 1999) that has no dynamical effect if the angular momentum is transported outward, but it is dynamically important in case the opposite is true. The global solution of ADAF showed that the flow actually becomes transonic at around few Schwarzschild radii $\left(r_{g}\right)$, and the self-similarity may be maintained far away from the sonic point (Chen et al. 1997).

Simultaneous with these developments, there were some interesting research going on regarding sub-Keplerian flows around black holes. It has been shown that sub-Keplerian flow does possess multiple sonic points in a significant range of the energy-angular momentum parameter space (Liang \& Thompson 1980). One of the consequences of the existence of multiple sonic points is that the flow accreting through the outer sonic point can be slowed down by the centrifugal barrier. This slowed-down matter acts as a barrier to the faster fluid following it. If the strength of the barrier is strong enough, then accretion shocks may form (Chakrabarti 1989). General global solutions in the advective domain incorporating viscosity and thermal effects were obtained by many independent researchers (Chakrabarti 1990, 1996; Lu et al. 1999; Lanzafame et al. 1998; Gu \& Lu 2004). Furthermore, it has also been shown that the global ADAF solution is a subset of the general advective solutions (Lu et al. 1999). Whether a flow will follow an ADAF solution or some kind of hybrid solution with or without shock will depend on the outer boundary condition and the physical processes dominant in the disk.

Although steady-state solutions are possible in a certain range of parameter space (Chakrabarti 1989; Chakrabarti \& Das 2004; Molteni et al. 1994, 1996a), advective solutions 
with discontinuities such as shocks are generally prone to various kinds of instabilities. Since various flow variables across the shock surface jump abruptly, this results in a markedly different cooling, heating, and other dissipation rates across the shock. This may render the shock unstable. For example, in the presence of bremsstrahlung cooling, resonance between cooling timescales and infall timescales in the post-shock part of the disk gives rise to oscillating shocks (Molteni et al. 1996b). Lanzafame et al. (1998) showed that beyond a critical viscosity, the post-shock disk may oscillate. The interaction between the outflow and the inflow may also cause the bending instability in the disk (Molteni et al. 2001). Molteni et al. (1999) showed that in the presence of non-axisymmetric azimuthal perturbations the shock initially becomes unstable, but stabilizes within a finite radial extent into an asymmetric closed pattern. Moreover, the post-shock region may be associated with the elusive Compton cloud that produces the hard photons (Chakrabarti \& Titarchuk 1995; Chakrabarti \& Mandal 2006; Mandal \& Chakarabarti 2008) and may also be the base of the jet (Das \& Chakrabarti 1999; Das et al. 2001; Chattopadhyay \& Das 2007; Das \& Chattopadhyay 2008; Becker et al. 2008; Das et al. 2009). Therefore, instabilities of the post-shock region may manifest itself as the variabilities observed in the emitted hard photons seen in microquasars and active-galactic nuclei (Molteni et al. 1996b). To add a new twist, Fukumura \& Tsuruta (2004) conjectured the presence of multiple shocks, and Giri et al. (2010) actually reported the presence of two oscillating shocks giving rise to two quasi-periodic oscillations (QPOs).

In this paper, we concentrate on the study of instabilities of rotating fluid around black holes, generated by the angular momentum transport by viscosity. Since the temperature, density, etc. are higher and the velocity is lower in the post-shock region compared to the pre-shock region, the angular momentum transport rate should be different in the two regions of the disk. In other words, in this paper we simulate transonic, viscous, rotating fluid around black holes. We employ a new code to study the effect of angular momentum transport in the accretion disk. Unlike other purely Eulerian codes, this new code is especially developed to strictly conserve angular momentum in the absence of viscosity. In Section 2, the governing equations and assumptions are presented. In Section 3, the code which was built to calculate the evolution of angular momentum as accurately as possible is described, along with tests for a rotating transonic flow and a viscous flow. In Section 4, the structure and the instability shown in simulations are presented, along with descriptions on the nature of the instability. The summary and discussion are presented in Section 5.

\section{BASIC EQUATIONS}

The one-dimensional time-dependent equations for quasispherical accretion of viscous flows are given by

$$
\begin{gathered}
\frac{\partial \rho}{\partial t}+\frac{1}{r^{2}} \frac{\partial}{\partial r}\left(r^{2} \rho v_{r}\right)=0 \\
\frac{\partial v_{r}}{\partial t}+v_{r} \frac{\partial v_{r}}{\partial r}+\frac{1}{\rho} \frac{\partial p}{\partial r}=\frac{l^{2}}{r^{3}}-\frac{\partial \Phi_{i}}{\partial r}, \\
\frac{\partial l}{\partial t}+v_{r} \frac{\partial l}{\partial r}=\frac{1}{r^{2} \rho} \frac{\partial}{\partial r}\left[\mu r^{4} \frac{\partial}{\partial r}\left(\frac{l}{r^{2}}\right)\right], \\
\frac{\partial e}{\partial t}+v_{r} \frac{\partial e}{\partial r}+\frac{p}{r^{2} \rho} \frac{\partial}{\partial r}\left(r^{2} v_{r}\right)=f \frac{\mu r^{2}}{\rho}\left[\frac{\partial}{\partial r}\left(\frac{l}{r^{2}}\right)\right]^{2},
\end{gathered}
$$

where $\rho, v_{r}, l, \Phi_{i}$, and $e$ are the gas density, radial velocity, specific angular momentum, gravitational potential, and specific internal energy, respectively. The angular velocity is defined as $\Omega=l / r^{2}$. The suffix $i$ in Equation (2) denotes $\mathrm{N}$ or $\mathrm{PN}$, corresponding to Newtonian or pseudo-Newtonian gravity (Paczyński \& Wiita 1980), respectively, and are given by

$$
\Phi_{\mathrm{N}}=-\frac{G M_{\mathrm{BH}}}{r}
$$

and

$$
\Phi_{\mathrm{PN}}=-\frac{G M_{\mathrm{BH}}}{r-r_{g}},
$$

where $M_{\mathrm{BH}}$ is the black hole mass and the Schwarzschild radius is $r_{g}=2 G M_{\mathrm{BH}} / c^{2}$. The pseudo-Newtonian potential is widely used to mimic the Schwarzschild geometry. For the gas pressure, the equation of state for ideal gas is assumed, i.e.,

$$
p=(\gamma-1) \rho e
$$

where $\gamma$ is the ratio of specific heats. For viscosity, the $\alpha$ prescription (Shakura \& Sunyaev 1973) can be assumed, i.e., the dynamical viscosity coefficient is described by

$$
\mu=\alpha \rho \frac{c_{s}^{2}}{\Omega_{K}},
$$

where

$$
c_{s}^{2}=\frac{\gamma p}{\rho}
$$

is the square of the adiabatic sound speed, and

$$
\Omega_{K}=\frac{l_{K}}{r^{2}}=\left[\frac{1}{r} \frac{d \Phi_{i}}{d r}\right]^{1 / 2}
$$

is the Keplerian angular velocity, and the viscosity parameter $\alpha$ is a constant which is generally less than 1 . Note that the actual expression of $\Omega_{K}$ depends on the gravitational potential used. Finally, following NY94, the parameter $f$ measures the fraction of the viscously generated energy that is stored as entropy and advected along with flows. The value $f=1$ corresponds to the limit of full advection and has been used in this paper.

In the following, we use $c$ and $r_{g}$ as the units of velocity and length, respectively, unless otherwise stated. In geometrical units, the unit of time is $\tau_{g}=r_{g} / c$.

\section{CODE AND TESTS}

One of the most demanding tasks in carrying out numerical simulations of Equations (1)-(4) is to calculate the evolution of the angular momentum as accurately as possible. Capturing shocks sharply should also be important in resolving structures with clarity, if shocks are involved. It has been known that the latter can be achieved by using codes based on modern, upwind finite-difference schemes on an Eulerian grid. However, without viscosity in such Eulerian codes, it is normally the azimuthal momentum $\left(\rho v_{\phi}\right)$ and not the angular momentum that is treated as a conserved quantity. On the other hand, codes based on the Lagrangian concept, such as the SPH code, can be designed to preserve the angular momentum strictly. Although it has been successfully applied to many studies of accretion flows, the SPH code is known to be unduly dissipative (see, e.g., Molteni et al. 1996a for discussions). 
Here, we describe an Eulerian code that was generated to accurately calculate the evolution of the angular momentum including its transport due to viscosity, and at the same time to capture discontinuities (shocks and contact discontinuities) sharply with minimum numerical dissipation. The code is composed of two parts: hydrodynamic and viscosity parts. The hydrodynamic part is based on the Lagrangian Total Variation Diminishing (TVD) plus remap approach. The Lagrangian/ remap approach is not new in numerical hydrodynamics and was employed previously (Colella \& Woodward 1984), but here we show that in this approach the equation for angular momentum conservation can be directly solved, and so the hydrodynamics part can be designed to preserve the angular momentum strictly in the absence of viscosity. At the same time, the TVD scheme (Harten 1983; Ryu et al. 1993) guarantees sharp reproductions of discontinuities and minimum numerical dissipation. In the viscosity part, the viscous angular momentum transfer is updated through an implicit method, assuring that it is free from numerical instabilities related to it. The viscous heating is updated with a second-order explicit method, since it is less subjected to numerical instabilities.

\subsection{Hydrodynamic Part}

The hydrodynamic part consists of the Lagrangian step and the remap step. First, in the Lagrangian step, the equations for Lagrangian hydrodynamics are solved. On the Lagrangian grid defined with mass coordinate, Equations (1)-(4), except for the centrifugal force, gravity, and viscosity terms which are treated separately (see below), can be written in a conservative form as

$$
\begin{gathered}
\frac{d \tau}{d t}-\frac{\partial\left(r^{2} v_{r}\right)}{\partial m}=0 \\
\frac{d v_{r}}{d t}+r^{2} \frac{\partial p}{\partial m}=0 \\
\frac{d l}{d t}=0 \\
\frac{d E}{d t}+\frac{\partial\left(r^{2} v_{r} p\right)}{\partial m}=0
\end{gathered}
$$

where $\tau$ and $E$ are the specific volume and the specific total energy, respectively, that are related to the quantities used in Equations (1)-(4) as

$$
\tau=\frac{1}{\rho}, \quad E=e+\frac{v_{r}^{2}}{2} .
$$

The mass coordinate is related to the spatial coordinate via

$$
d m=\rho(r) r^{2} d r
$$

and its position can be followed with

$$
\frac{d r}{d t}=v_{r}(m, t)
$$

Equations (11), (12), and (14) form a hyperbolic system of conservation equations, and upwind schemes can be applied to build codes that advance the Lagrangian step using Harten's TVD scheme, which is an explicit, second-order, finite-difference scheme to solve a hyperbolic system of conservation equations
(Harten 1983; Ryu et al. 1993). We note that the angular momentum in Equation (13) is preserved, so it need not be updated in the Lagrangian step.

In the remap step, the quantities evolved in the Lagrangian grid are redistributed to the Eulerian grid to preserve the spatially fixed grid structure. Before the Lagrangian step, the Lagrangian and Eulerian grid zones coincide. But after the step, the Lagrangian grid zone moves to the updated position

$$
r^{\text {new }}=r^{\text {old }}+\bar{v} \Delta t
$$

where $\bar{v}$ is the time-averaged velocity, and so it no longer coincides with the Eulerian grid zone. Not only are the quantities of the density, radial momentum, and total energy conserved in the Eulerian grid, but the angular momentum is also remapped. For the remap, we employ the third-order accurate scheme used in the PPM code (see Colella \& Woodward 1984 for details).

With the Lagrangian and remap steps, Equations (1)-(4) are updated in the Eulerian grid, except for the centrifugal force, gravity, and viscosity terms on the right-hand side. The centrifugal force and gravity terms are calculated separately after the Lagrangian and remap steps such that

$$
v_{i}^{\text {hydro }}=v_{i}^{\text {lag+remap }}+\Delta t\left(\frac{l_{i}^{\text {remap }}}{r_{i}^{3}}-\left.\frac{d \Phi}{d r}\right|_{i}\right) .
$$

Then, the viscosity terms are calculated, as discussed in the following subsection.

Non-uniform Eulerian grids can be employed in the code. For the problem in this paper, we use a grid, where the size of cells increases exponentially as

$$
\Delta r_{i}=\Delta r_{1} \times \delta^{i-1}
$$

to achieve higher resolution at the origin. Here, $\Delta r_{1}$ is the size of the first grid cell and $\delta$ is the increment factor.

\subsection{Viscosity Part}

Viscosity has two effects on accretion flows. First, it transfers the angular momentum outward, allowing the matter to accrete inward. At the same time, it acts as friction, which results in viscous heating.

Since the term for the angular momentum transfer in Equation (3) is linear in $l$, it can be solved implicitly. Substituting $\left(l^{\text {new }}+l^{\text {remap }}\right) / 2$ for $l$, Equation (3) without the advection term becomes

$a_{i} l_{i-1}^{\text {new }}+b_{i} l_{i}^{\text {new }}+c_{i} l_{i+1}^{\text {new }}=-a_{i} l_{i-1}^{\text {remap }}-\left(b_{i}-2\right) l_{i}^{\text {remap }}-c_{i} l_{i+1}^{\text {remap }}$,

forming a tridiagonal matrix. Here, $a_{i}, b_{i}$, and $c_{i}$ are given with $\rho, \mu$, and $r$ as well as $\Delta r$ and $\Delta t$. The tridiagonal matrix can be solved easily for $l^{\text {new }}$ with an appropriate boundary condition (Press et al. 1992). The term for the viscous heating in Equation (4) is also linear in $e$ (note that $\mu \propto e$ ), so it can be solved implicitly, too. However, combining the two linear equations for $l$ and $e$ becomes complicated. Through numerical experiments, we found that the explicit treatment for the viscous heating does not cause any numerical problem. So, instead of implementing a complicated scheme to solve simultaneously $l$ and $e$ implicitly, we solve the angular momentum transfer implicitly, while solving the viscous heating explicitly. 


\subsection{Tests}

Two tests are presented to demonstrate that the code can handle transonic flow as well as viscous flow, both of which are involved in our problem. The capability of the code to capture shocks sharply and resolve structures clearly is tested with a transonic accretion flow. In these tests we reproduce well-known results.

The evolution of an inviscid flow, which enters the outer boundary with a small amount of angular momentum and approaches a black hole described by Paczyński \& Wiita potential (Paczyński \& Wiita 1980), is calculated in cylindrical geometry. We note that previous subsections describe the code only in spherical geometry, but the code is actually written in arbitrary geometries. For this test, the version in the cylindrical geometry is used. Without viscosity, the angular momentum is preserved. A shock can form, if the rotating flow through the outer sonic point approaches the centrifugal barrier and decelerates discontinuously, due to the twin effect of centrifugal force and pressure. In the test, $l=1.8 \mathrm{cr}_{g}$, a value slightly below the marginally stable value $\left(l_{\mathrm{ms}}=(3 / 2)^{3 / 2} c r_{g}\right)$, is used. The values of the flow quantities in geometrical units are $\left(\rho, p, v_{r}\right)=(0.71809,0.007604,-0.083566)$ at the injection radius $r_{\text {inj }}=50 r_{g}$. The sound speed at $r_{\text {inj }}$ is $c_{s}=0.1188 c$; hence, the fluid is subsonically injected. The fluid becomes supersonic after crossing the outer sonic point at $r_{\text {co }}=27.9 r_{g}$, becomes subsonic at the shock $r_{\mathrm{sh}}=7.89 r_{\mathrm{g}}$, and enters the black hole supersonically after crossing the inner sonic point at $r_{\mathrm{ci}}=2.563 r_{g}$. For the ratio of specific heats, $\gamma=4 / 3$ is used. This is the case of a stable standing shock considered in Molteni et al. (1996a). In Figure 1, the numerical solutions (open circles) of density $\rho$ (top) and radial Mach number $M_{r}=v_{r} / c_{s}$ (bottom) using 2048 uniform grid cells are compared with the analytical solution (solid line). The flow in smooth regions coincides with the analytical solution very well and the shock position matches the analytical value very well. The agreement of the analytical result with the current code is better than those with the purely Eulerian TVD code and the SPH code presented in Molteni et al. (1996a).

Next, the performance of the code for a subsonic viscous flow is tested with a self-similar ADAF solution. Matter is steadily injected with Keplerian angular velocity into the computational domain at $r_{\text {inj }}$, and the simulation lasts until the steady state is reached. Figure 2 presents the flow quantities after the steady state is reached and compares them with the analytic solution. The Newtonian potential is used, and the ADAF is described with a self-similar solution (NY94). The values of the physical parameters used in this test are $\gamma=4 / 3$, and $\alpha=0.3$. The simulation was performed on an exponentially increasing grid of 780 cells with $\Delta r_{1}=0.4972 r_{s}$ and $\delta=1.01$. Here, $r_{s}$ is the sink size. The injection position is $r_{\text {inj }} \sim 3.6915 \times 10^{4} r_{s}$. The quantities are drawn in units of the Keplerian velocity and the Keplerian angular momentum at the sink, $v_{K}\left(r_{s}\right)$ and $l_{K}\left(r_{s}\right)$, and the density is in an arbitrary unit. The figure shows that the analytic solution is reproduced very closely in the region between $r \sim 10 r_{s}$ and $r \sim 10^{4} r_{s}$ in a box of size $1.1611 \times 10^{5} r_{s}$. The error in the specific angular momentum is less than a few percent at most.

\section{RESULTS OF SIMULATIONS}

In previous numerical works, oscillation phenomena in accretion flows around black holes related to the QPO were reported. The study of inviscid supersonic accretion flows around
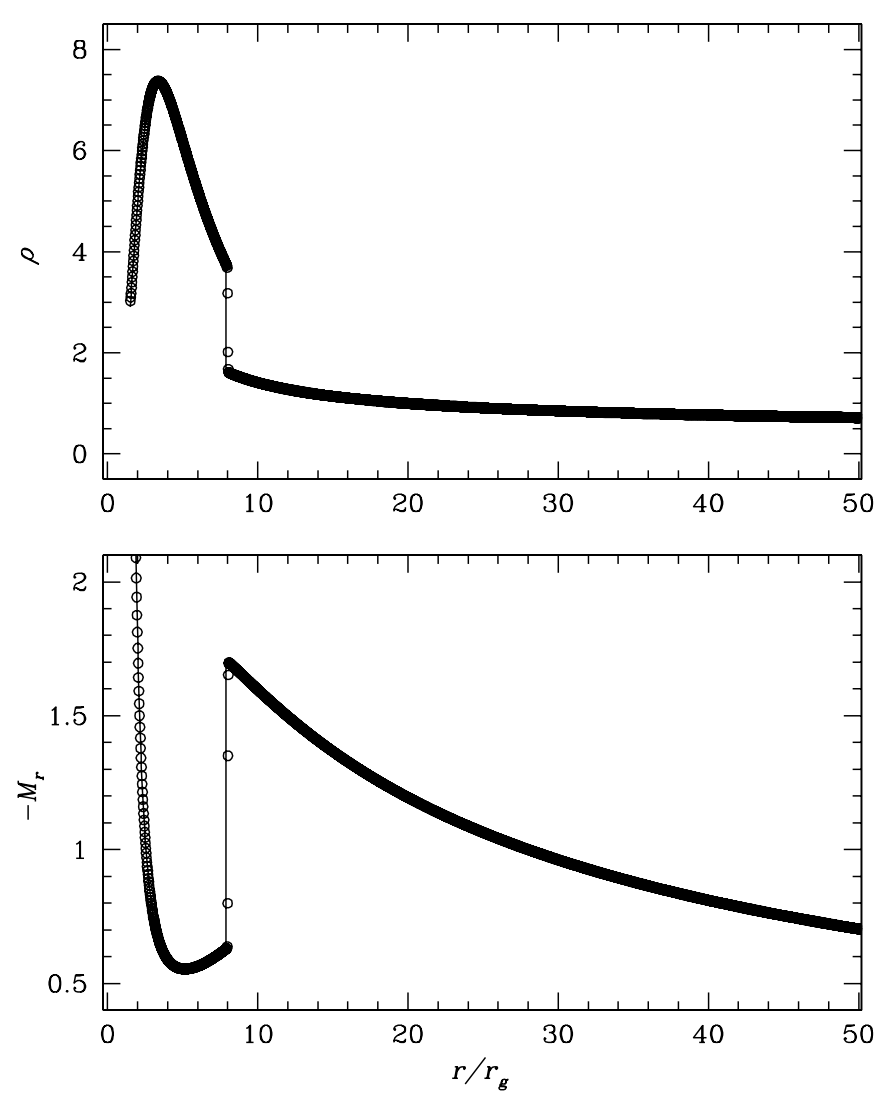

Figure 1. Comparison of numerical solution with analytic solution for a onedimensional accretion flow in cylindrical geometry that allows a standing shock. The plots show the density $(\rho)$ and the radial Mach number $\left(M_{r}\right)$. The solid curves represent the analytical solution and the open circles represent the numerical solution with 2048 uniform grid cells.

a Newtonian central object showed the accretion disk with shock structure to be dynamically unstable (Ryu et al. 1995). Global transonic accretion flows around black holes have been known to exhibit stationary shocks for inviscid (Chakrabarti 1989; Molteni et al. 1996a; Das et al. 2001) as well as dissipative flows (Chakrabarti 1996; Lu et al. 1999; Lanzafame et al. 1998; Becker et al. 2008). However, since the post-shock flow is hotter, denser, and slower, the dissipation rate in the post-shock flow is shorter than that of the pre-shock flow, which may make the post-shock flow unstable. Indeed, it has been shown that the energy-angular momentum parameter space for standing shock decreases with the increase of viscosity parameter (Chakrabarti \& Das 2004; Gu \& Lu 2004; Das et al. 2009). Lanzafame et al. (1998) simulated viscous transonic flow and showed that steady shocks exist for low viscosity, while for higher viscosity the shock becomes unstable. However, Lanzafame et al. (1998) restricted their investigations for a hot flow $\left(T_{\text {inj }} \sim 10^{11} \mathrm{~K}\right.$ at the injection radius), and very low viscosity parameter $(\alpha \lesssim$ few $\times$ $10^{-3}$ ). Since the pre-shock flow was chosen to be hot (postshock disk was obviously even hotter), the angular momentum removal was very efficient in both the pre-shock and post-shock disks, even when the viscosity parameter was low. The length scale of the computation box was only about a few tens of $r_{g}$. In this paper, we simulate viscous transonic flow which is cold to begin with, and investigate the instability arising from reasonably higher viscosity of the flow. The reason to choose cold flow at the injection is to have a very different angular momentum transport rate in the post-shock and pre-shock disks, and thereby to maximize the effect of shock instability. Moreover, 

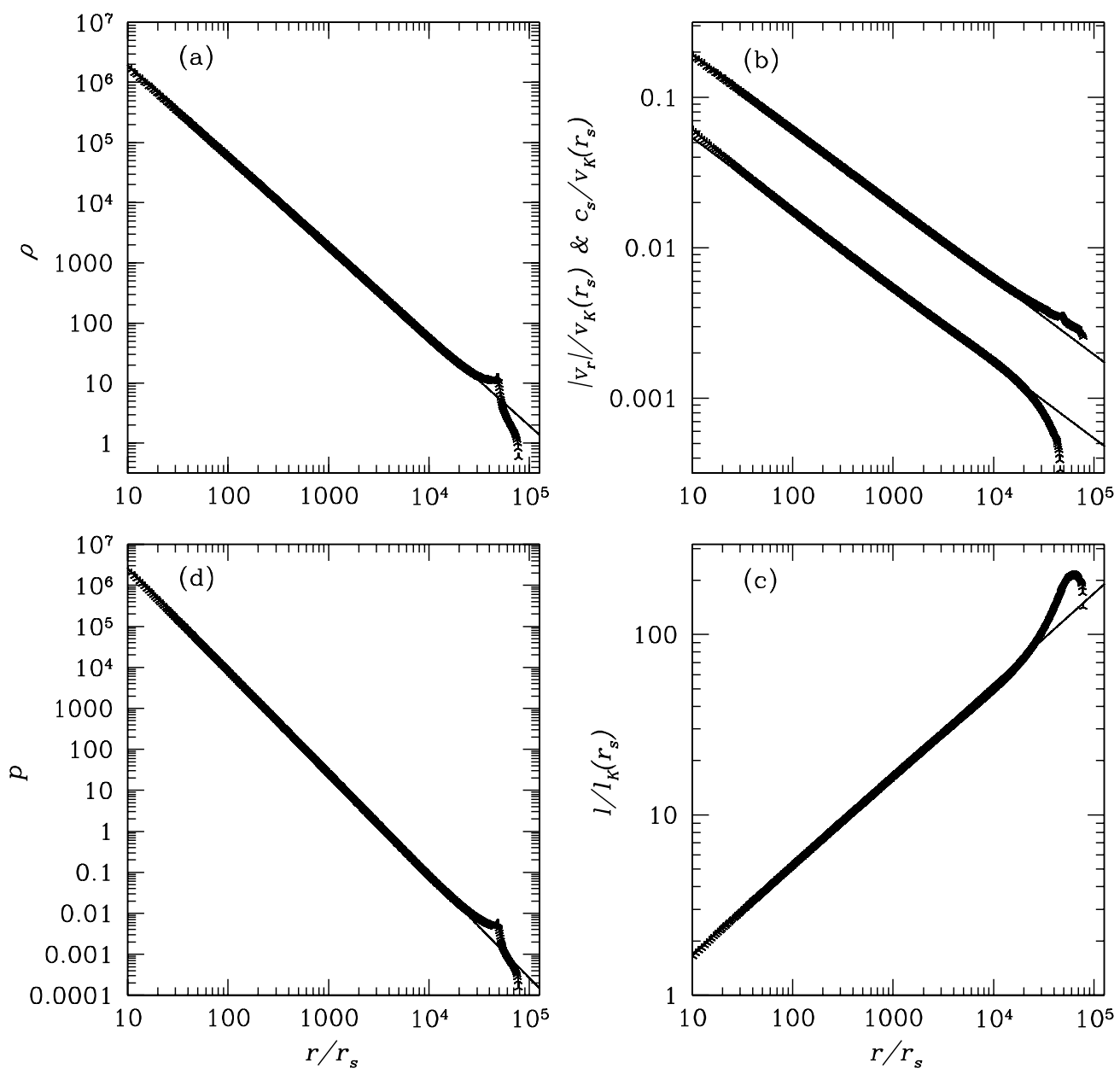

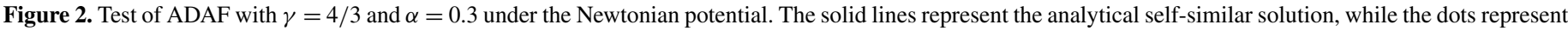

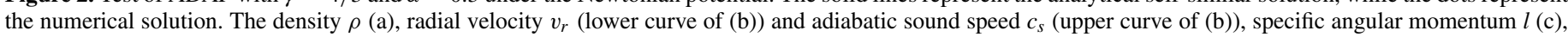
and pressure $p(\mathrm{~d})$ are shown clockwise.

we keep the length scale of the computation box fairly large so as to study large amplitude and low frequency shock instability.

\subsection{Shock Formation in Inviscid Rotating Flow}

We start our set of simulations with a low energy, rotating, transonic, inviscid flow around a black hole (described by $\Phi_{\mathrm{PN}}$ ). The steady-state, inviscid, transonic solution corresponds to a flow characterized by the Bernoulli parameter or specific energy (which in our unit system is $\mathcal{E}=0.5 v_{r}^{2}+c_{s}^{2} /(\gamma-1)+$ $\left.l^{2} /\left(2 r^{2}\right)-1 /\{2(r-1)\}\right)$ and specific angular momentum $(l)$. Parameters $\mathcal{E}, l$ for the inviscid flow are $1.25 \times 10^{-6} c^{2}$ and $1.8 c r_{g}$, respectively. The ratio of specific heat is given by $\gamma=1.4$. The Bondi radius (the length scale within which gravity becomes important) is defined as $r_{B}=G M_{\mathrm{BH}} / c_{s, \infty}^{2}$, where $c_{s, \infty}^{2}=\mathcal{E}(\gamma-1)$ for an inviscid flow. In this particular case, $r_{B}=10^{6} r_{g}$ and $c_{s, \infty}=7.071 \times 10^{-4} c$. The analytical, steady-state solution of flows for these parameters gives two physical sonic points, the inner one is at $r_{\mathrm{ci}}=2.394 r_{g}$ and the outer one at $r_{\mathrm{co}}=199991.04 r_{g} \approx 0.2 r_{B}$. The analytical solution also predicts a shock at $r_{\mathrm{sh}}=22.2 r_{g}$. It has been shown in connection to Figure 1 that it is possible to simulate transonic flow quite accurately with subsonic injection, i.e., when $r_{\text {inj }}>r_{\text {co }}$. However, in the present scenario, $r_{\text {sh }} \ll r_{\text {co. }}$. Therefore, if $r_{\text {inj }}>r_{\mathrm{co}}$, then a large amount of computation time will be wasted in simulating the uninteresting region of the disk. Hence, without any loss of generality, we choose the injection parameters from the supersonic portion of the analytical curve in order to reduce computation time. To further reduce the computation time and also to achieve higher resolution close to the center, we use exponentially increasing grids, which have 3553 cells with $\Delta r_{1}=0.0296$ and $\delta=1.001$, and the length of the computation box corresponds to $1000 r_{g}=0.001 r_{B}$. The injection radius is hence $r_{\mathrm{inj}}=1000 r_{g}=0.001 r_{B}$, and the flow radial velocity $\left(v_{r}(\mathrm{inj})=2.970 \times 10^{-2} c\right)$, specific angular momen$\operatorname{tum}\left(l_{\text {inj }}=1.8 c r_{g}\right)$ and sound speed $\left(c_{s}(\right.$ inj $\left.)=4.827 \times 10^{-3} c\right)$ at $r_{\text {inj }}$ is taken from the analytical solution. In Figure 3, we compare the steady-state analytical solution (solid line) with the simulation result (open circles) when the steady state is reached. Various flow variables such as $\rho$ (a), $v_{r}(\mathrm{~b}), c_{s}(\mathrm{c})$, and $p(\mathrm{~d})$ are plotted with $\log (r)$. Figure 3 shows excellent agreement of the simulation result with the analytical curve and the shock captured within two to three cells.

\subsection{Shock Oscillation of Viscous Flow}

Time-dependent solutions of viscous transonic accretion flow are obtained by starting with the inviscid flow described in Section 4.1 as the initial condition, and then increasing the viscosity parameter $\alpha$. The action of the viscosity can be understood from Equations (3) and (4). For accretion (i.e., when $v_{r}<0$ ), if the right-hand side of Equation (3) is negative, then the angular momentum will be transported outward. In 


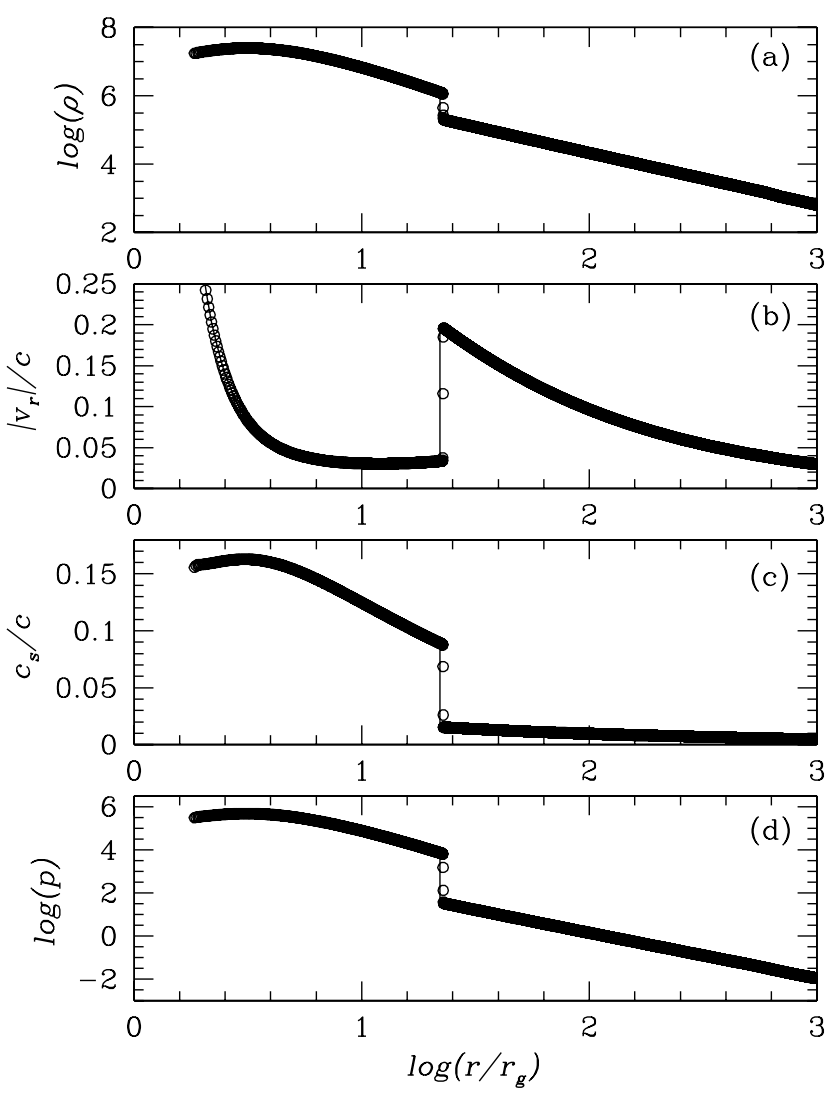

Figure 3. Comparison of the analytical (solid line) with the numerical solution (open circles) of hydrodynamical accretion shock for transonic flow with $\gamma=1.4$ and $l=1.8 c r_{g}$. The injection radius $r_{\text {inj }}=1000 r_{g}$, and at $r_{\text {inj }}$ the radial velocity $v_{r}($ inj $)=2.970 \times 10^{-2} c$ and sound speed $c_{s}($ inj $)=4.827 \times 10^{-3} c$.

the presence of Shakura-Sunyaev-type viscosity, the angular momentum transport in the post-shock subsonic flow is much more efficient than the pre-shock supersonic flow. As a result of which, angular momentum starts to pile up in the immediate post-shock fluid, resulting in a jump in the angular momentum distribution across the shock. Similarly, Equation (4) tells us that the viscous heat dissipation in the post-shock disk will also be higher compared to the pre-shock disk.

It is well known that a standing shock forms if the total pressure (ram+gas pressure) is conserved across the shock (Chakrabarti 1989). In Figure 4(a), the inviscid solution produces a stationary shock at location $r_{\mathrm{sh}}=22.2 r_{g}$, as is shown in Figure 3. In Figure 4(b), the shock location as a function of $t$ is plotted for $\alpha=0.003$. The excess gas pressure due to viscous heat dissipation and the increased centrifugal force due to the piled-up angular momentum in the post-shock disk push the shock front outward. For low $\alpha$, the shock front moves to a larger location $\left(r_{\mathrm{sh}} \sim 31 r_{g}\right.$ as in Figure 4(b)) where the balance between the total outward push and the total inward pressure from the pre-shock flow is restored. However, for higher viscosity parameter $\alpha=0.006$ (Figure 4(c)), the enhanced angular momentum transport creates an even stronger outward push and the shock front overshoots a possible equilibrium position and the shock starts to oscillate. Interestingly, when the shock moves to around $\sim 70 r_{g}$ and beyond, a second shock tends to emerge, which expands and collides with the outer shock. The combined shock then drifts outward, the inner shock re-emerges, and the cycle continues. In the following, let us perform a detailed investigation on transonic flow with higher viscosity and the emergence of two shocks.
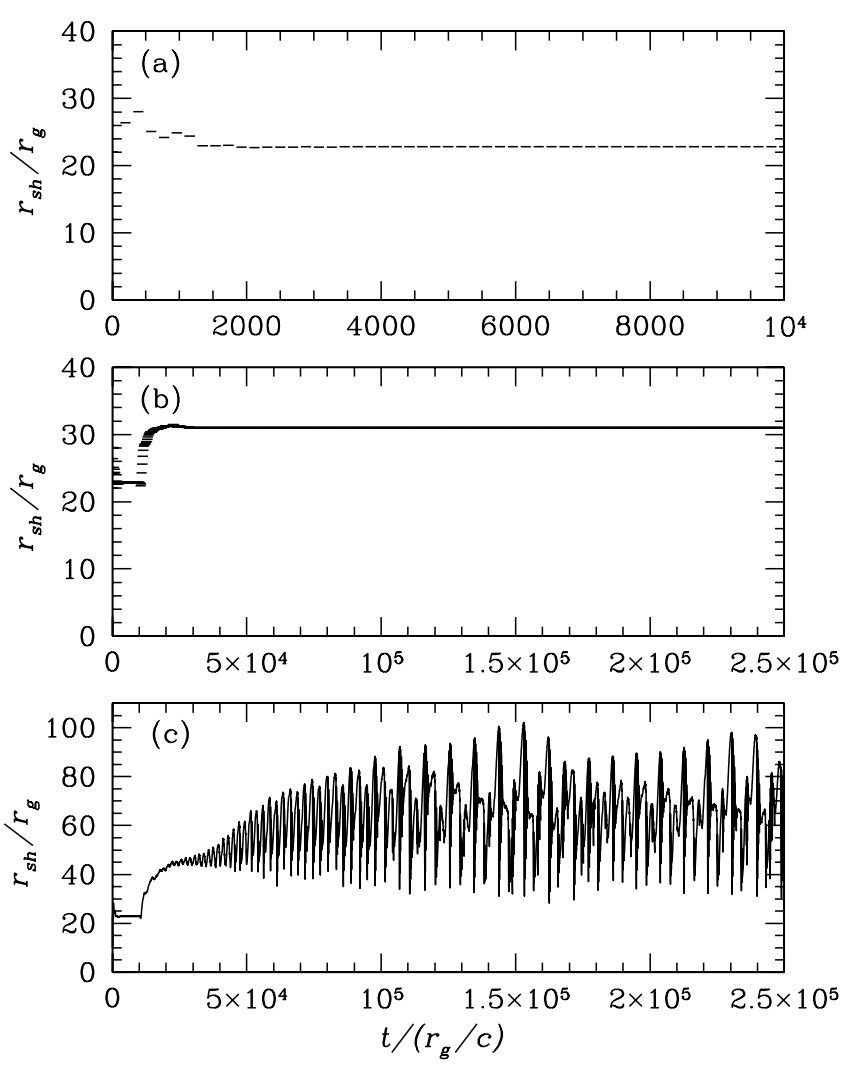

Figure 4. Comparison of the shock location $r_{s h}$ for $\alpha=0.0$ (a), $\alpha=0.003$ (b) and $\alpha=0.006$ (c). The injection radius is $r_{\text {inj }}=1000 r_{g}$, the injected radial velocity $v_{r}($ inj $)=2.970 \times 10^{-2} c$, sound speed $c_{s}($ inj $)=4.827 \times 10^{-3} c$, and angular momentum $l_{\text {inj }}=1.8 c r_{g}$. The adiabatic index is $\gamma=1.4$.

In Figures 5(a)-(d), we have plotted the radial velocity $v_{r}$ (dashed-dot) and the sound speed $c_{s}$ (solid) at four time steps: (a) $t=2.9 \times 10^{5} r_{g} / c$, (b) $t=3.165 \times 10^{5} r_{g} / c$, (c) $t=3.4 \times 10^{5} r_{g} / c$, and (d) $t=3.615 \times 10^{5} r_{g} / c$, where the outer boundary conditions are same as in Figure 3 , and $\alpha=0.01$. In Figures 6(a)-(d), the specific angular momentum distribution $l(r)$ is plotted for the same simulation and for the same time steps as in Figures 5(a)-(d). The corresponding panels of Figures 5 and 6 are to be considered in tandem to understand this complicated phenomenon. The different snapshots in Figures 5(a)-6(d), correspond to (1) the maxima in outer shock (Figures 5(a) and 6(a)), (2) the expansion stage of the combined shock just after the minima in the next cycle (Figures 5(b) and 6(b)), (3) just before the maxima of the outer shock (Figures 5(c) and 6(c)), and (4) just after the maxima of the outer shock (Figures 5(d) and 6(d)). In Figure 5(a), there are two shock structures, the inner shock is at $r_{\text {sh }}($ in $) \sim 130 r_{g}$ and the outer shock is at $r_{\mathrm{sh}}$ (out) $\sim 500 r_{g}$. The corresponding angular momentum distribution in Figure 6(a) shows that the $d l / d r>0$ in the range $r<20 r_{g}$ and $r_{\mathrm{sh}}$ (in) $<r<r_{\mathrm{sh}}$ (out), while $d l / d r<0$ is in the range $20 r_{g}<r<r_{\text {sh }}$ (in). In Figure 5(b), the two shocks merge and the combined shock is at $r_{\mathrm{sh}}($ in $)=r_{\mathrm{sh}}$ (out) $=r_{\mathrm{sh}} \sim 100 r_{g}$. Figure 6(b) shows that $d l / d r>0$ in a region where $r<20 r_{g}$, and $d l / d r<0$ for $20 r_{g}<r \lesssim r_{\mathrm{sh}}$, with a smaller hump in angular momentum distribution around $r_{\mathrm{sh}}$. The angular momentum distribution attains a tall peak, and the enhanced centrifugal pressure almost stalls the infall (Figure 5(b)) in that region. However, due to the extra pressure from the piled-up $l$ the combined shock moves outward, while the contact discontinuity wave resulting from 


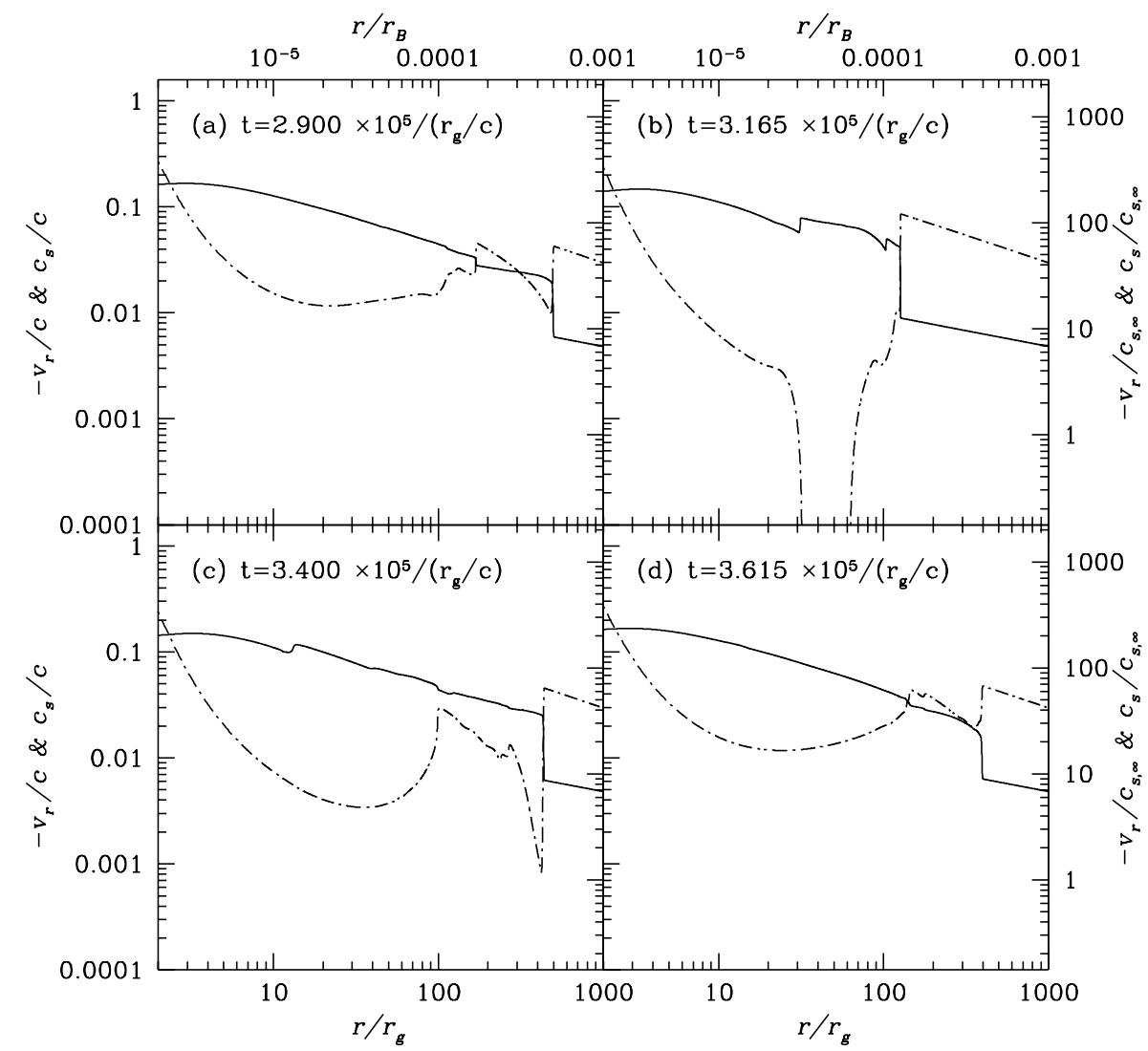

Figure 5. Four snapshots of radial velocity (dashed-dot) and sound speed (solid) of a viscous fluid of $\alpha=0.01$ and $\gamma=1.4$. The injection parameters are $r_{\text {inj }}=1000 r_{g}$, $l_{\text {inj }}=1.8 c r_{g}, v_{r}($ inj $)=2.970 \times 10^{-2} c$, and $c_{s}($ inj $)=4.827 \times 10^{-3} c$. The time sequence goes as $(\mathrm{a}) \rightarrow(\mathrm{b}) \rightarrow(\mathrm{c}) \rightarrow(\mathrm{d})$.

the collision of shocks moves toward the black hole. As a result, the sound speed (i.e., temperature) in the immediate post-shock flow drops (Figure 5(c)), and the angular momentum distribution becomes $d l / d r>0$ (Figure 6(c)). This allows for a freer infall and $v_{r}$ in the immediate post-shock disk increases considerably (Figure 5(c)). As the contact discontinuity wave is absorbed by the black hole, the angular momentum in the immediate post-shock region gets reduced considerably, and $v_{r}$ becomes supersonic in the region. However, the flow again hits the centrifugal barrier closer to the black hole and the inner shock re-emerges (Figures 5(d) and 6(d)). We note that the regions of $d l / d r<0$ are subject to rotational instability. The non-steady behavior shown here should be partly attributed to the instability.

\subsubsection{On Emergence of the Inner Shock, Shock Collision, and the Angular Momentum Transfer}

In the top panel of Figure 7, the shock oscillation is plotted for $\alpha=0.01$. Therefore, each panel of Figures 5(a)-(d) and $6(\mathrm{a})-(\mathrm{d})$ corresponds to the various snapshots of flow variables taken from the top panel of Figure 7 (time sequences (a)-(d) are marked in the figure). Similar to Figure 4(c), $r_{\mathrm{sh}}$ in the top panel of Figure 7 starts to oscillate as the viscosity is turned on. A transient inner shock, i.e., $r_{\mathrm{sh}}$ (in), develops when $r_{\mathrm{sh}}($ out $) \gtrsim 80 r_{g}$. Initially, the dynamics of the two shocks are similar to that of Figure 4(c), i.e., the inner shock forms when $r_{\mathrm{sh}}($ out $)$ is at the maxima, and then $r_{\mathrm{sh}}(\mathrm{in})$ collides with the contracting outer shock, and the merged shock then expands. However, for $t>0.2 \times 10^{5} \tau_{g}$, the shock dynamics slowly change; both shocks expand and then contract, and the shocks collide while contracting. The merged shock then reaches a minimum and then expands, and this cycle continues. The query about the formation of the inner shock can be understood as follows: as the original shock expands to a distance $\gtrsim 80 r_{g}$, the sound speed in the immediate post-shock region and close to the black hole differs by almost an order of magnitude. Hence, the rate of angular momentum transport in a region closer to the inner sonic point is much higher than the region closer to the shock. Hence, the angular momentum transport rate is not only markedly different between the post-shock and pre-shock regions, but also within the post-shock flow when the shock expands to a very large distance. Hence, the angular momentum piles up in between the inner sonic point $\left(r_{\mathrm{ci}}\right)$ and the shock (e.g., Figure 6(b)), which enhances the centrifugal barrier and impedes the accretion. Continued shock expansion reduces the post-shock sound speed (i.e., temperature) and creates a mild but positive angular momentum gradient, which increases the infall velocity in the immediate post-shock flow. This can continue up to the extent that the post-shock fluid once again becomes supersonic in the immediate post-shock domain; however, further downstream the piled-up angular momentum virtually stops the supersonic inflow causing the formation of the inner shock. The inner shock again increases the temperature, which causes the inner shock to expand, too. If the outer shock is contracting then the two shocks may collide, or both shocks may expand in phase and collide during the contraction phase. The combined shock then expands and the whole cycle is repeated. It may be noted that the inner shock emerges halfway into each of the cycles and hence it is a persistent feature.

It is interesting to seek the radiative property of such oscillatory dynamics of the disk. We estimate the bremsstrahlung loss a posteriori from the disk as representative of the radiative loss. 


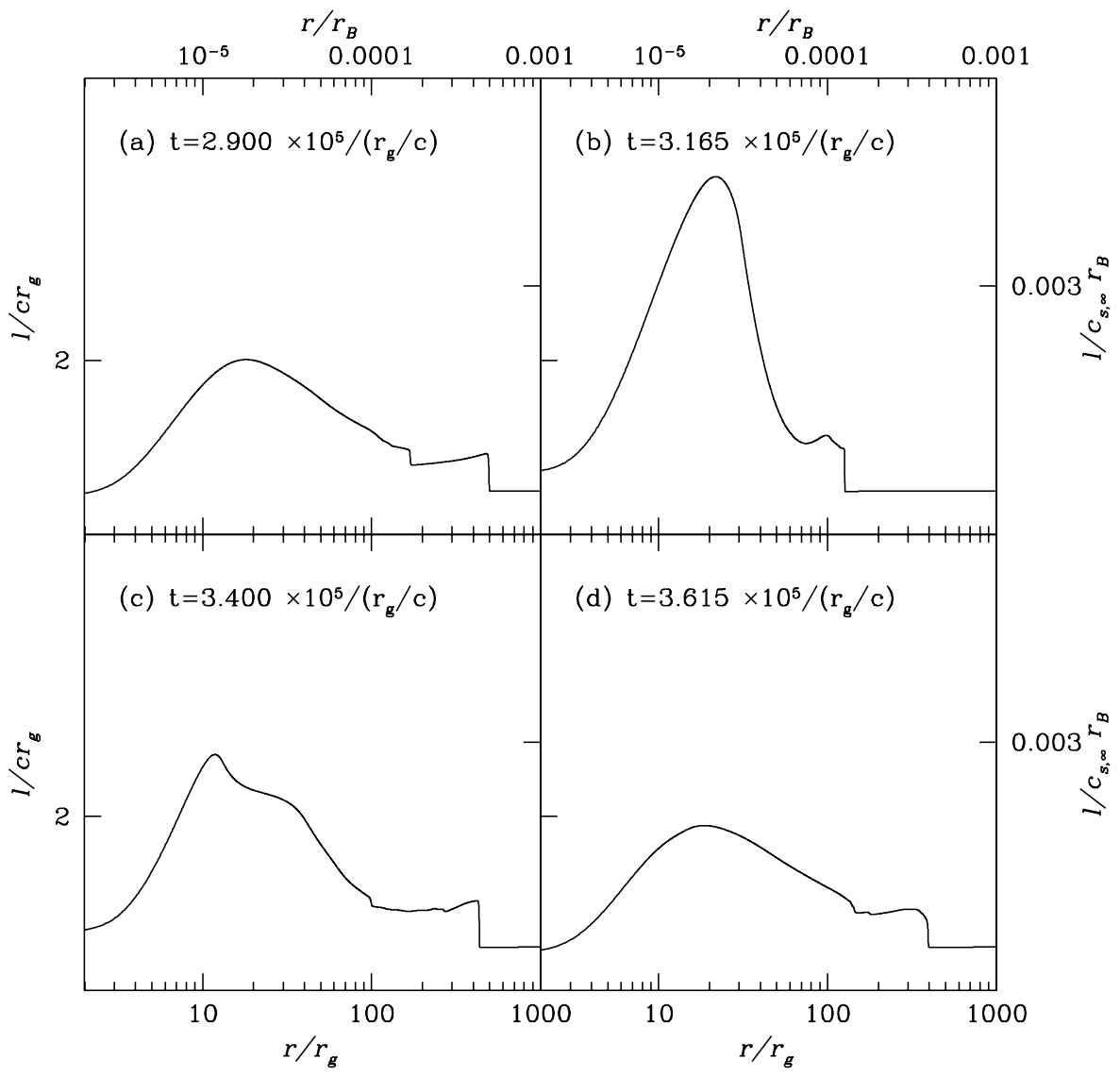

Figure 6. Specific angular momentum at the four different snapshots in the same simulation as in Figure 5 . The time sequence goes as (a) $\rightarrow$ (b) $\rightarrow($ c) $\rightarrow(d)$.

It is well known that the bremsstrahlung emission $\propto \rho^{2} c_{s}$. In Figure $7(\mathrm{~b})$, we plot $E_{\text {Total Br }} / \rho_{\mathrm{inj}}^{2} c_{s}$ (inj) as a function of time, where

$$
E_{\text {Total Br }}=\int_{r_{\text {sink }}}^{r_{\text {inj }}} \rho^{2} c_{s} r^{2} d r
$$

$\rho_{\mathrm{inj}}$ and $c_{s}$ (inj) are the density and sound speed of the flow at the outer boundary of $r_{\text {inj }}$. Hence, the bottom panel of Figure 7 represents the total bremsstrahlung emission from the computational box compared to the bremsstrahlung emission at $r_{\text {inj }}$. Interestingly, bremsstrahlung emission also has a periodic behavior, whose period is similar to the period of the shock oscillation. However, the shock maxima/minima may or may not coincide with either emission maxima or minima. In this particular case, there is initially no distinct correlation, but as the oscillation approaches quasi-saturation, the emission maxima coincide with the combined shock minima, and the emission minima coincide with the rising phase of the combined shock and when the inner shock has not been formed. As the combined shock contracts it pushes the post-shock matter inward just like a "bellow" in a blacksmith's shop. This increases the $\rho, c_{s}$, and $v_{r}$. The enhanced $\rho$ and $c_{s}$ contribute to form the emission maxima. As the combined shock expands, the flow variables like $\rho, c_{s}$ in the immediate post-shock region decreases, and the emission starts to decrease until it reaches the minima. Since the wide difference of $c_{s}$ also triggers the differential $l$ transfer in the inner regions and outer regions of the post-shock disk, the angular momentum again starts to pile up and starts the formation of the inner shock described above. There exists a secondary peak in the bremsstrahlung emission as well which appears to be related to the dynamics of the inner shock. The time lag between the shock maxima and the emission maxima is $\delta t \sim 2 \times 10^{4} \tau_{g}$. Initially, the oscillation period of the shock was $T_{\text {osc }}^{\prime} \sim 5 \times 10^{3} \tau_{g}$. The oscillation period gradually increases to a quasi-saturation value of $T_{\text {osc }} \sim 8 \times 10^{4} \tau_{g}$. Since

$$
\tau_{g}=\frac{2 G M}{c^{3}} \sim 10^{-5} \frac{M_{\mathrm{BH}}}{M_{\odot}} \mathrm{s},
$$

therefore,

$$
T_{\mathrm{osc}} \sim 8 \times 10^{4} \tau_{g} \sim 8 \times 10^{-1} \frac{M_{\mathrm{BH}}}{M_{\odot}} \mathrm{s} .
$$

This would correspond to the frequency of $\sim 0.125 \mathrm{~Hz}$ for a stellar mass black hole i.e., $M_{\mathrm{BH}} \sim 10 M_{\odot}$. In the case of a supermassive black hole $\left(M_{\mathrm{BH}} \sim 10^{8} M_{\odot}\right)$, these timescales will correspond to $2.5 \mathrm{yr}$ variabilities. However, since there are two shocks, we are interested to see the influence of the dynamics of the two shocks on emission. In the left panel of Figure 8, we plot the outer shock (top), inner shock (middle), and the relative bremsstrahlung emission (bottom) for reference, and in the right panels we plot the power density spectra for the outer shock (top), inner shock (middle), and the bremsstrahlung emission (bottom) for a stellar mass black hole $\left(M_{\mathrm{BH}}=10 M_{\odot}\right)$. The power density spectrum of the outer shock shows a frequency of $\sim 0.125 \mathrm{~Hz}$. The power density spectrum of the inner shock has a prominent peak at the frequency $\sim 0.25 \mathrm{~Hz}$ and a weaker peak around $\sim 0.125 \mathrm{~Hz}$, the secondary peak suggests that the oscillation of the outer shock forces a weak periodicity on the inner shock as well. The power density spectrum of the inner shock is a bit noisy, since the time variation of the inner shock, 
although persistent, is not continuous. Interestingly, Figure 8, shows that the bremsstrahlung emission also peaks at around the same frequencies as that of the two shocks, confirming that the quasi-periodicity in the emission is due to the quasi-periodicity of the two shocks.

In the presence of such a dynamical disk, it is intriguing to investigate the time variation of the amount of matter and angular momentum consumed by the black hole. Let us define the massloss parameter, or the ratio of the rate of mass cannibalized by the black hole to the rate of mass injected, as $\dot{M} / \dot{M}_{\mathrm{inj}}$, where $\dot{M}=\left.\left(\rho v_{r} r^{2}\right)\right|_{\text {sink }}$ and $\dot{M}_{\text {inj }}=\left.\left(\rho v_{r} r^{2}\right)\right|_{r_{\text {inj }}}$. The angular momentum loss rate is defined as $\dot{L} / \dot{L}_{\text {inj }}$, where $\dot{L}=\dot{M} l_{\text {sink }}$ and $\dot{L}_{\text {inj }}=\dot{M}_{\text {inj }} l_{\text {inj. }}$. The average specific angular momentum of the disk is defined as

$$
\langle l\rangle=\frac{\int l d r}{\int d r} .
$$

In Figure 9, we plot the mass-loss parameter (top panel), angular momentum loss rate (middle), and the average angular momentum of the disk (bottom) as a function of time. The profiles of the mass-loss parameter and the angular momentum loss rate are similar to that of the bremsstrahlung emission rate. Since the distribution of $\rho$ peaks when the shock is at the minima, the peaks of the mass-loss parameter and the angular momentum loss rate coincide with the peak of the bremsstrahlung emission. As the shock recedes, $v_{r}$ and $\rho$ decrease, resulting in matter accumulating in the disk i.e., $\dot{M} / \dot{M}_{\text {inj }}<1$. As the shock contracts, it squeezes more matter into the black hole (accumulated in the expansion phase) than it is being supplied; therefore
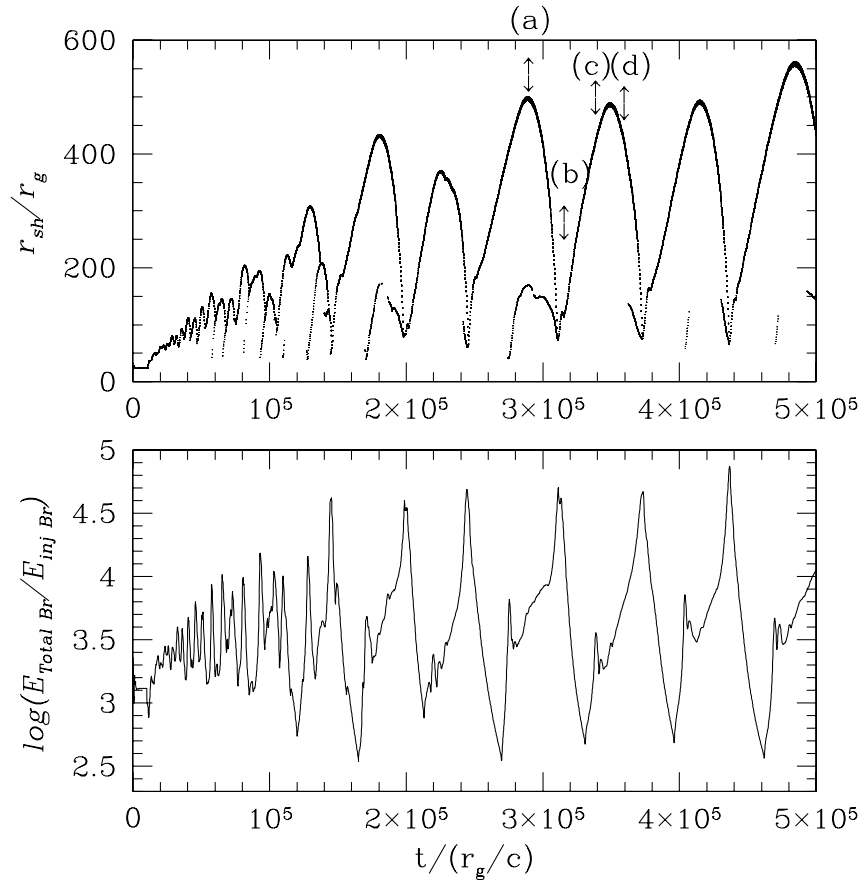

Figure 7. Shock position (top) and bremsstrahlung emission (bottom) as a function of time in the same simulation as in Figure 5. The upper curve in the top panel is the outer shock and the lower curve is the inner shock. The snapshots of time in Figures 5(a)-(d) are marked on the top panel as (a)-(d).
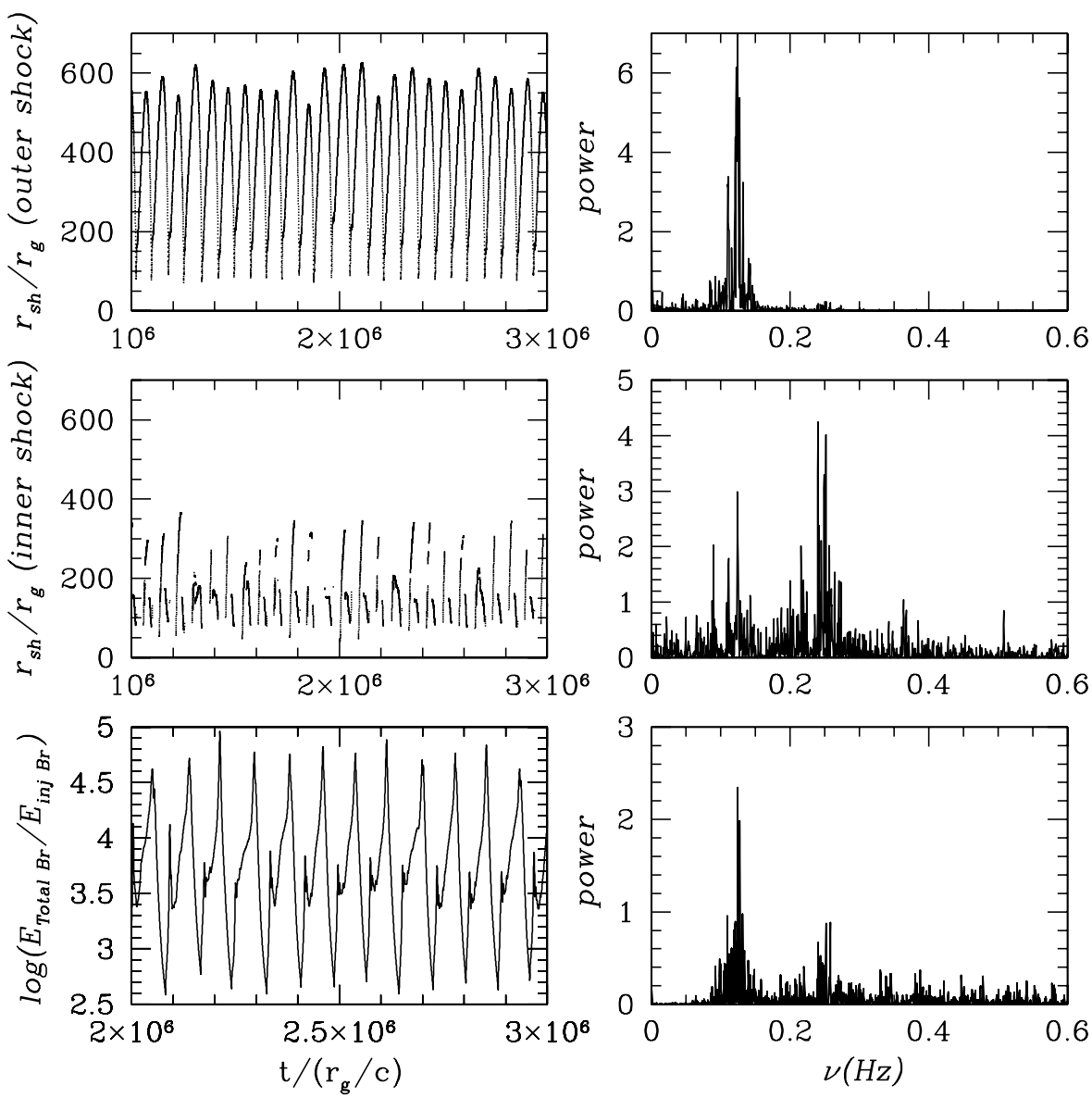

Figure 8. Left panels: outer shock (top), inner shock (middle), and the bremsstrahlung emission (bottom) as a function on of time. Right panels: the power density spectra of the outer shock (top), inner shock (middle), and the bremsstrahlung emission (bottom). Same simulation as in Figure 5. 

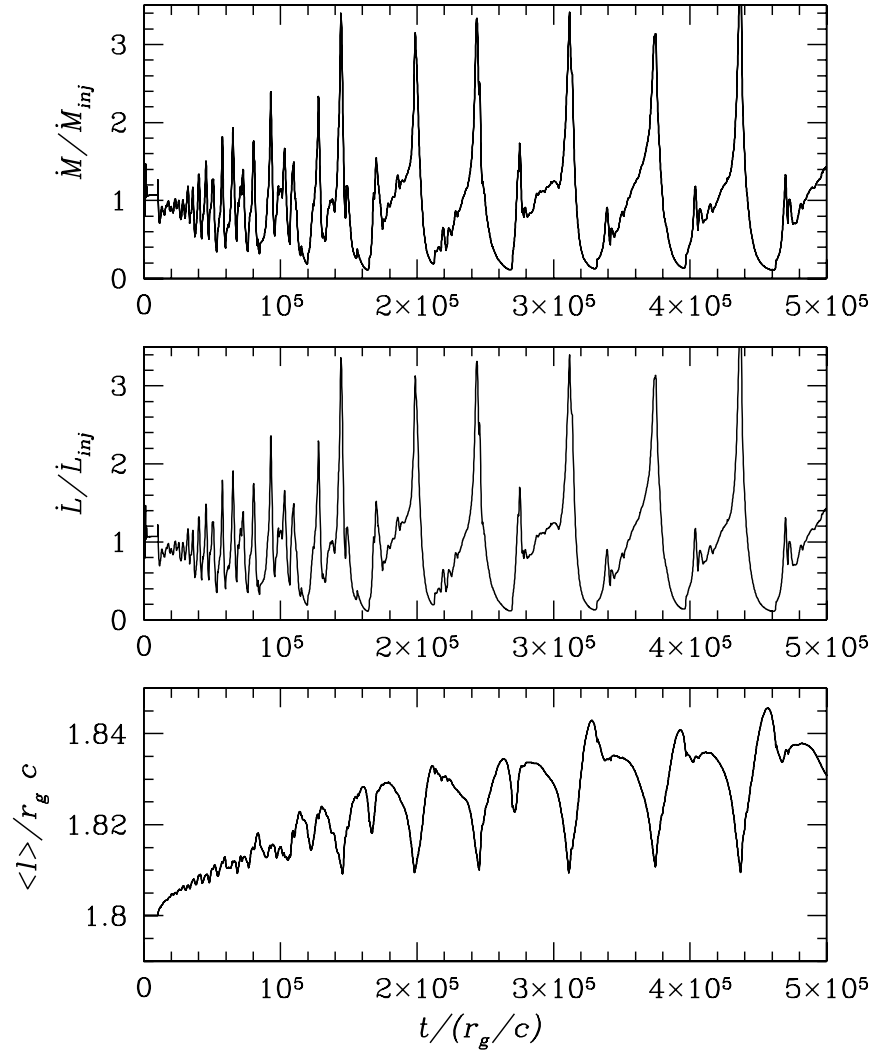

Figure 9. Mass accretion rate (top), angular momentum loss rate (middle), and averaged specific angular momentum (bottom) in the post-shock region of the outer shock as a function of time in the same simulation as in Figure 5.

$\dot{M} / \dot{M}_{\text {inj }}>1$. It is to be noted that in this particular case, the disk prefers to stay in the state where $\dot{M} / \dot{M}_{\text {inj }} \lesssim 1$. The angular momentum loss rate follows $\dot{M} / \dot{M}_{\text {inj }}$. Interestingly, the maxima of the average angular momentum of the disk coincide with the minima of the emission, mass-loss rate, and the angular momentum loss rate. The bottom panel of Figure 9 suggests that if the average angular momentum of the disk increases, then $v_{r}$ should decrease in a large region of the disk, which should reduce the rate of matter actually accreted onto the black hole. The average angular momentum $(\langle l\rangle)$ of the disk increases with the increase of the peak and the width of the angular momentum distribution of the disk, which corresponds to the dips in the emission, mass-loss parameter, and angular momentum loss rate. Although $\langle l\rangle$ oscillates with the same period as that of the shock, the disk surprisingly prefers to stay in state $\langle l\rangle$, which is greater than $l_{\text {inj }}$. Since the disk itself is oscillating, all these flow parameters should oscillate with the same period. And indeed, the bremsstrahlung emission, the mass-loss rate, the angular momentum loss rate, etc. all oscillate with the same period of shock oscillation.

\subsubsection{Shock Oscillation for Higher Viscosity}

The dynamics of the disk with a higher viscosity parameter is different from that due to the lower one. For the higher viscosity parameter, the difference in the disk dynamics will arise from more efficient angular momentum transfer as well as higher viscous dissipation of heat, even if the outer boundary condition remains the same. In Figure 10, we have plotted the shock location with time (top) and the bremsstrahlung emission with time (bottom) for a fluid with the same injection parameters as
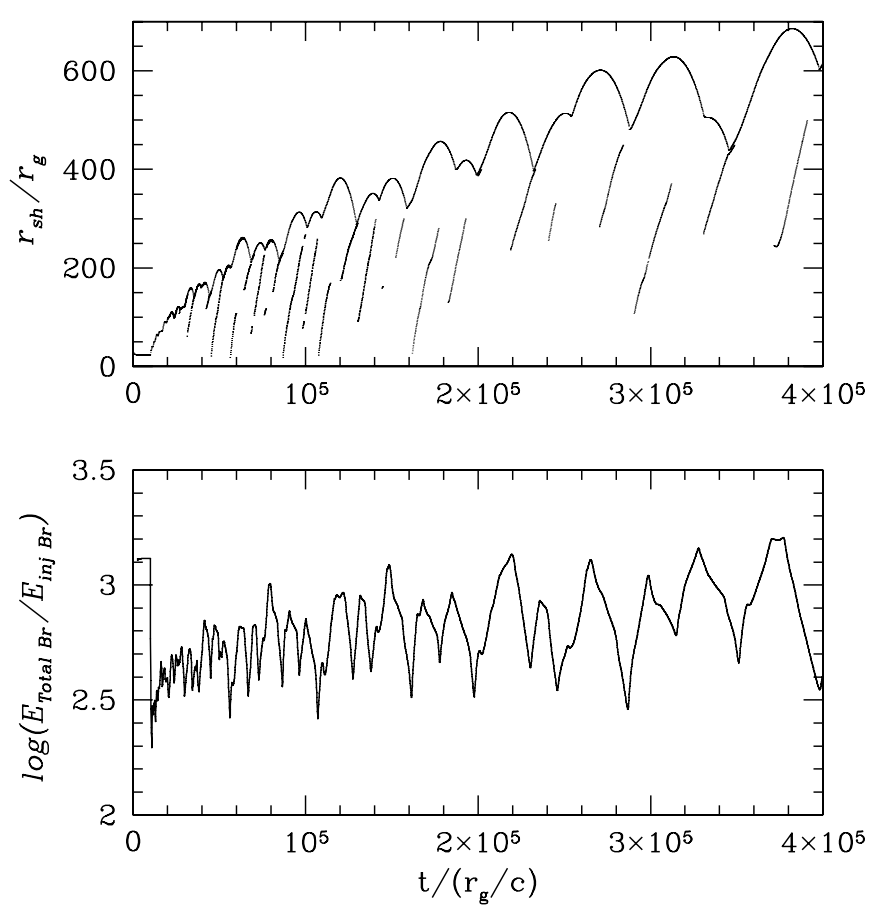

Figure 10. Shock position (top) and bremsstrahlung emission (bottom) as a function of time. The upper curve in the top panel is the outer shock and the lower curve is the inner shock. The simulation is for $\alpha=0.1$ and $\gamma=1.4$, and injection parameters $r_{\text {inj }}=1000 r_{g}, l_{\text {inj }}=1.8 c r_{g}, v_{r}$ (inj) $=2.970 \times 10^{-2} c$, and $c_{s}($ inj $)=4.827 \times 10^{-3} c$.

the inviscid flow described in Section 4.1, i.e., $r_{\text {inj }}=1000 r_{g}$, $v_{r}($ inj $)=2.970 \times 10^{-2} c$, and $c_{s}$ (inj) $=4.827 \times 10^{-3} c$, and the viscosity parameter is $\alpha=0.1$. The time variation of the shock for $\alpha=0.1$ (Figure 10) is distinctly different from that of the shock for $\alpha=0.01$ (i.e., Figure 7). The inner shock forms, expands, and at some epoch collides with the contracting outer shock, while at some other epoch it disappears before colliding with the outer shock. The inner shock is weaker compared to the disk with lower $\alpha$. The time evolution of the two shocks is somewhat similar to the initial phases of the shock variation for $\alpha=0.01$. Comparison of the time variation of the bremsstrahlung emission with the time variation of the shock shows no correlation between shock minima and emission maxima unlike the case for $\alpha=0.01$. In Figures 11(a)-(b), $v_{r}$ (dashed-dot) and $c_{s}$ (solid) are plotted corresponding to the emission maxima (Figure 11(a)) and emission minima (Figure 11(b)). Similarly, the corresponding specific angular momentum distributions are plotted for the emission maxima (Figure 11(c)) and minima (Figure 11(d)), and the densities too are plotted for the emission maxima (Figure 11(e)) and minima (Figure 11(f)). The maxima of the bremsstrahlung emission occur when the inner shock is tending to form, while the minima occur when the inner shock has not been formed (also refer to Figure 10). This change in the behavior of the shock and the emission properties compared to that of the $\alpha=0.01$ actually depends on the different rates of angular momentum transfer. Since the viscosity in the present case is tenfold higher than $\alpha=0.01$, the outward angular momentum transport is very efficient. So, close to the black hole, the angular momentum rises steeply outward unlike the flow with lower viscosity (e.g., Figures 11(c)-(d) may be compared with Figures 6(a)-(d)). If the shock is closer to the black hole, then the peak of the angular momentum distribution is very close to the outer 


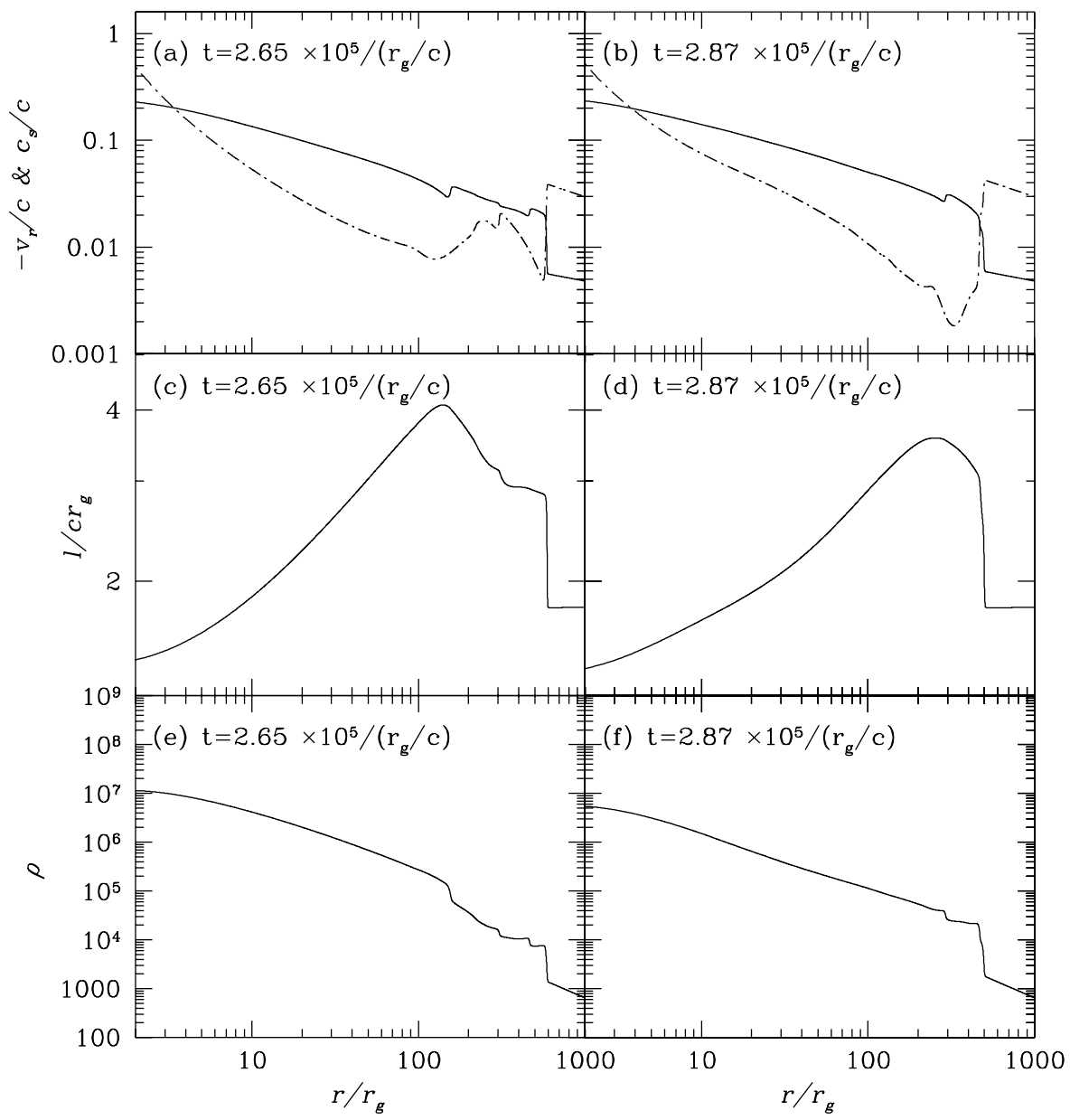

Figure 11. Radial velocity (dashed-dot) and sound speed (solid) of viscous fluid for time $t=2.65 \times 10^{5} \tau_{g}$ (a) and $t=2.87 \times 10^{5} \tau_{g}$ (b). Specific angular momentum is plotted for time $t=2.65 \times 10^{5} \tau_{g}$ (c) and $t=2.87 \times 10^{5} \tau_{g}$ (d). Density in arbitrary units is plotted for $t=2.65 \times 10^{5} \tau_{g}$ (e) and $t=2.87 \times 10^{5} \tau_{g}$ (f). The simulation is for $\alpha=0.1$ and $\gamma=1.4$. The injection parameters are $r_{\text {inj }}=1000 r_{g}, l_{\text {inj }}=1.8 c r_{g}, v_{r}(\mathrm{inj})=2.970 \times 10^{-2} c$, and $c_{s}($ inj $)=4.827 \times 10^{-3} c$.

shock (Figure 11(d)); this causes matter to accrete more freely between the horizon and the peak of the angular momentum distribution, and hence the density is lower (Figures 11(b), (d), and (f)). This causes the emission to dip. As the shock moves out, the angular momentum peak is situated farther inside (Figure 11(c)). This causes the matter to madly fall inward between the outer shock and the inner $l$ peak. As the infalling matter encounters the angular momentum pile-up, it decelerates drastically, increasing the density considerably; and hence, the bremsstrahlung emission is enhanced (Figures 11(a), (c), and (e)). Eventually, it forms an inner shock, but the enhanced energy deposition in the post-inner shock region causes the inner shock to expand, thereby reducing density and emission. In this connection, one may point out that the immediate post-shock (for both inner and outer shocks) region may be decelerating or accelerating (e.g., Figures 5(a)-(d), 11(a)-(b)). However, it was predicted by Nakayama (1992) and Nobuta \& Hanawa (1994) that post-shock acceleration and deceleration correspond to unstable and stable shocks, respectively. The reason for this is that no standing shock can exist in the viscous flow for the corresponding initial conditions.

In the top panel of Figure 12 , we plot $\dot{M} / \dot{M}_{\text {inj }}$, and like the lower viscosity case, its peak and trough coincide with that of the bremsstrahlung emission. The angular momentum loss rate $\dot{L} / \dot{L}_{\text {inj }}$ (middle) also follows the pattern of $\dot{M} / \dot{M}_{\text {inj }}$. Since the angular momentum distribution is higher during the peak emission, the average angular momentum of the disk's $\langle l\rangle$ (bottom) peak coincides with the emission peak. Moreover, $\dot{M} / \dot{M}_{\text {inj }}<1$ most of the time, which means that because of higher angular momentum most of the matter being injected into the disk is not being consumed by the black hole, which is indicated by the fact that $\langle l\rangle$ is significantly higher than $l_{\text {inj }}$.

The role of viscosity can be ascertained if one compares the viscous time with the advection timescale. The viscous timescale may be defined as

$$
\tau_{\mathrm{vis}}=\int_{r_{\mathrm{ci}}}^{r_{\mathrm{sh}}} \frac{r}{v} d r,
$$

where $\nu=\mu / \rho$ and the advection timescale

$$
\tau_{\mathrm{ad}}=\int_{r_{\mathrm{ci}}}^{r_{\mathrm{sh}}} \frac{d r}{\left|v_{r}\right|} .
$$

The time variation of $\tau_{\mathrm{vis}}$ closely follows the corresponding variation of the shock location, with minima and maxima of each, coinciding with the other at exactly the same time. When compared to the $\alpha=0.01$ case, at the shock minima, $\tau_{\text {vis }}$ and $\tau_{\text {ad }}$ are comparable and we see that the shock front is pushed outward. At the shock maxima $\tau_{\text {ad }} \ll \tau_{\text {vis }}$, i.e., advection dominates; consequently, the shock front hurls inward contracting significantly from few $\times 100 r_{g}$ to few $\times 10 r_{g}$. 

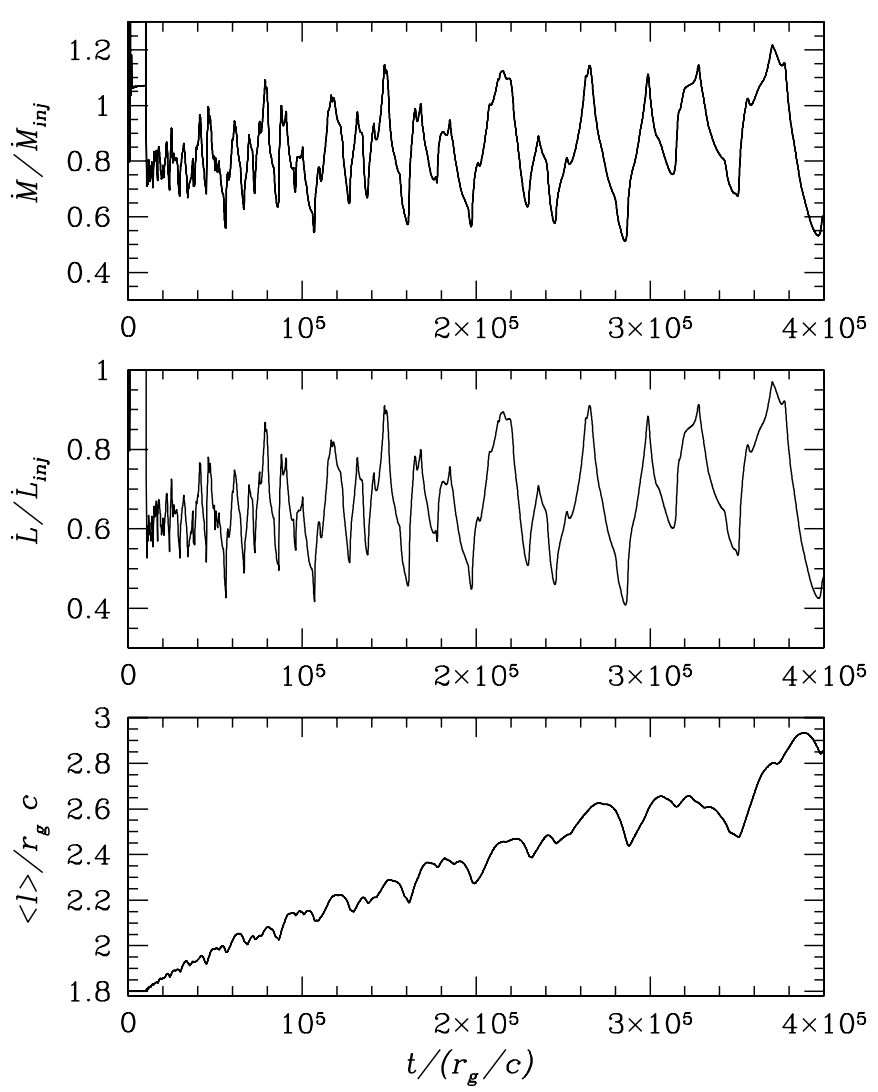

Figure 12. Mass accretion rate (top), angular momentum loss rate (middle), and averaged specific angular momentum (bottom) in the post-shock region of the outer shock as a function of time in the same simulation as in Figure 10.

Hence, the interplay between these two timescales sustains the oscillation. The general pattern of the temporal behavior of the timescales and the relation to the shock oscillation for the flow with $\alpha=0.1$ is similar to that with the flow $\alpha=0.01$. However, $\tau_{\mathrm{ad}}$ and $\tau_{\mathrm{vis}}$ for $\alpha=0.1$ are roughly comparable and hence oscillations do not saturate. Foglizzo \& Tagger (2000) investigated for Bondi flow that entropy-acoustic cycles may sustain shock oscillation if $c_{s}\left(r_{\mathrm{ci}}\right) / c_{s}\left(r_{\mathrm{sh}}\right) \gg 1$. In the simulations we have run, $\max \left[c_{s}\left(r_{\mathrm{ci}}\right) / c_{s}\left(r_{\mathrm{sh}}\right)\right] \sim 10$, but generally the ratio is less in most of the times. Hence, the effect of entropy-acoustic cycles in regulating shock oscillation is probably moderate in our case. A multi-dimensional simulation may give a more definitive answer.

\section{SUMMARY AND DISCUSSION}

This paper intended to study the time-dependent simulations of large amplitude oscillations of advective, viscous, subKeplerian disks, to complement earlier works studying low amplitude oscillations undertaken by Molteni and his collaborators (Lanzafame et al. 1998). As an improvement, we have employed a new code which uses the Lagrangian TVD/remap approach. This code strictly conserved the angular momentum without viscosity and reduced the numerical dissipation considerably (e.g., Section 3). Tests showed that the shock-capturing capability of this code is better than both standard Eulerian code and Lagrangian SPH code (e.g., Figure 1), and followed the angular momentum transfer of the viscous, subsonic, analytical solution extremely well (e.g., Figure 2).

Oscillation of the accretion shock was borne out by the different rates of angular momentum transfer across the shock and the heat dissipated due to the presence of viscosity. It has been shown that in the presence of the low viscosity parameter $(\alpha=0.003)$, the shock front of a disk, with the same initial and boundary conditions as those of the inviscid case, tended to expand and settled at a larger distance from the disk (Figure 4(b)). For an even higher viscosity $(\alpha \gtrsim 0.005)$, the rate of angular momentum transfer was higher, which caused a faster rate of shock front expansion. As the shock front exceeded a possible equilibrium position, it started to oscillate (Figure 4(c)). However, remember that the value of the critical viscosity parameter ( $\alpha \sim 0.005$ in the present case) is not sacrosanct, but actually depends on the initial condition. For example, it has been shown that the critical viscosity parameter will be higher for flows with lower angular momentum, while for a fluid with higher initial energy the critical viscosity parameter will be lower (see Chakrabarti \& Das 2004). Hence, if the proper initial condition is used then a stable shock is expected to form for higher viscosity parameters (i.e., $\alpha \sim 0.1-0.2$ ), too, the investigation of which, however, is not the point of interest for the present paper.

A detailed study of the disk dynamics was conducted for reasonably high viscosity (i.e., $\alpha=0.01$ and 0.1 ). For $\alpha=$ 0.01 , the shock oscillation amplitude was found to be quite high, $\gtrsim 100 r_{g}$. This resulted in a large sound speed gradient in the post-shock subsonic flow. In the case of large amplitude shock oscillation, the rate of outward angular momentum transport in a region closer to the inner sonic point was shown to be much higher compared to the rate of angular momentum transport near the shock. As a result, our simulation showed the angular momentum to be piled up in an intermediate region between the shock and the inner sonic point. The expanding shock also increased the inflow velocity in the immediate post-shock region, only to be decelerated by the extra centrifugal pressure due to the piled-up angular momentum further inside the disk (e.g., Figures 6(a)-(d)). The inflow velocity in the post-shock disk may be increased to the extent that it may again become supersonic, then the resistance from the excess centrifugal pressure from the piled-up angular momentum distribution may cause the formation of the inner shock. In the case of moderately high $\alpha$, the distance between the peak of the angular momentum distribution and the outer shock is large enough to allow for the $v_{r}$ to become supersonic again and enhanced the possibility of forming the inner shock. Note that the amplitude of shock oscillation will possibly be lesser for multi-dimensional simulations. Viscosity is more active in the post-shock disk, and hence the extra centrifugal force due to the piled-up angular momentum and the heat dissipated by viscosity both actively take part in shock oscillation. However, in the case of realistic accretion flow, part of the viscous heat dissipated in the postshock disk will also be spent to puff it up, which would imply less outward push on the shock surface. Hence, for a flow with the same injection and viscosity parameters, the oscillation amplitude for a multi-dimensional disk is expected to be less compared to a purely conical flow. Consequently, the critical viscosity above which the disk becomes oscillatory will also be higher.

The time evolution for shocks of higher viscosity was shown to be distinctly different from that of shocks with lower viscosity. The inner shock was weaker and more sporadic for a disk with $\alpha=0.1$. The main reason for this was the higher rate of angular momentum transport. Even when the shock was around $100 r_{g}$, highly efficient angular momentum transport created a smooth increase of angular momentum, which only peaked closer to 
$r_{\text {sh }}$. As the shock expanded, $v_{r}$ increased, but the opportunity of becoming supersonic was minimized since the peak of the $l(r)$ was closer to the shock. Hence, the inner shock, if it formed at all, was weaker. However, since the shock amplitude for $\alpha=0.1$ was much larger than the case with $\alpha=0.01$, in time the formation of the inner shock became more regular, and the behavior was more similar to that of $\alpha=0.01$.

The oscillatory motion of the shock induced oscillation in all the disk parameters such as emission, rate of matter consumed by the black hole, the rate of angular momentum consumed by the black hole, and the average angular momentum of disk. All these parameters oscillated with the same period as that of the shock. The disk oscillation started with $\alpha \gtrsim 0.005$. Considering $M_{\mathrm{BH}}=10 M_{\odot}$, for $\alpha=0.005$ the oscillation frequency of the outer shock was $5 \mathrm{~Hz}$ and that of the inner shock was $10 \mathrm{~Hz}$; for $\alpha=0.006$ the frequencies were $1 \mathrm{~Hz}$ and $3 \mathrm{~Hz}$, respectively, for the outer and inner shocks, and for $\alpha=0.01$ the two frequencies were $0.125 \mathrm{~Hz}$ and $0.25 \mathrm{~Hz}$. Hence, one may conclude that apart from the dependence of the oscillation frequency on injection parameters, the QPO frequency definitely decreases with increasing viscosity and vice versa. Observationally, GRO J1655-40 exhibits a rise in QPO frequency in its rising state and a fall in QPO frequency in its declining phase in 2005 (Chakrabarti et al. 2008). Chakrabarti et al. (2009) plotted the QPO frequency for the object XTE J1550-564 in 1998 burst phase. They showed that in the rising phase of the outburst, the low frequency QPO increases from $0.08 \mathrm{~Hz}$ to $13.1 \mathrm{~Hz}$ and then starts to decrease in the declining phase before disappearing. Such rise and fall of QPO frequencies may be explained by the change in shock oscillation frequency due to the change of the net viscosity of the disk.

In the presence of viscosity, a positive angular momentum gradient i.e., $d l / d r \geqslant 0$ helps in the outward transport of angular momentum. However, a negative gradient may trigger inward transport of angular momentum. The $d l / d r<0$ condition was attained in the disk in at least two locations, at the outer shock front and just behind the peak of the specific angular momentum distribution. Those regions were subject to rotational instability. $d l / d r<0$ caused the average angular momentum $\langle l\rangle$ of the disk to increase, and hence the period and the amplitude of the shock oscillation to increase, too. This is less perceptible for lower $\alpha$ and the shock oscillation achieved quasi-saturation, but for $\alpha=0.1$ the shock went outside the computation domain. We repeated the simulation with $\alpha=0.3$ (not presented in the paper) and in this case too the shock went outside the domain, although formation of the inner sonic point and oscillation of the two shocks were observed, too.

In the case of multi-dimensional simulations, a part of the post-shock matter would have ejected along the vertical direction in the form of winds, which would have carried away a part of the angular momentum, such that the increase of $\langle l\rangle$ may have been arrested for higher $\alpha$. This would have meant that the shock oscillation may saturate for $\alpha \geqslant 0.1$. Hence, we conjecture that the non-saturation of shock oscillation for $\alpha \gtrsim 0.1$ could be an artifact of one-dimensional simulation. We will test it in a future work using multi-dimensional simulations.

S.L. was supported in part by Basic Science Research Program through the National Research Foundation of Korea (NRF) funded by the Ministry of Education, Science and Technology (2010-0004738). D.R. was supported in part by National Research Foundation of Korea through grant KRF2007-341-C00020.

\section{REFERENCES}

Becker, P. A., Das, S., \& Le, T. 2008, ApJ, 677, L93

Chakrabarti, S. K. 1989, ApJ, 347, 365

Chakrabarti, S. K. 1990, MNRAS, 243, 610

Chakrabarti, S. K. 1996, ApJ, 464, 664

Chakrabarti, S. K., \& Das, S. 2004, MNRAS, 349, 649

Chakrabarti, S. K., Debnath, D., Nandi, A., \& Pal, P. S. 2008, A\&A, 489, 41

Chakrabarti, S. K., Dutta, B., \& Pal, P. S. 2009, MNRAS, 394, 1463

Chakrabarti, S. K., \& Mandal, S. 2006, ApJ, 642, L49

Chakrabarti, S. K., \& Titarchuk, L. 1995, ApJ, 455, 623

Chattopadhyay, I., \& Das, S. 2007, New Astron., 12, 454

Chen, X., Abramowicz, M., \& Lasota, J.-P. 1997, ApJ, 476, 61

Colella, P., \& Woodward, P. R. 1984, J. Comput. Phys., 54, 174

Das, S., Becker, P. A., \& Le, T. 2009, ApJ, 702, 649

Das, S., Chattopadhyay, I., \& Chakrabarti, S. K. 2001, ApJ, 557, 983

Das, S., \& Chattopadhyay, I. 2008, New Astron., 13, 549

Das, T. K., \& Chakrabarti, S. K. 1999, Class. Quantum Grav., 16, 3879

Foglizzo, T., \& Tager, M. 2000, A\&A, 363, 174

Fukumura, K., \& Tsuruta, S. 2004, ApJ, 611, 964

Giri, K., Chakrabarti, S. K., Samanta, M., \& Ryu, D. 2010, MNRAS, 403, 516

Gu, W. M., \& Lu, J. F. 2004, Chin. Phys. Lett., 21, 2551

Harten, A. 1983, J. Comput. Phys., 49, 357

Ichimaru, S. 1977, ApJ, 214, 840

Igumenshchev, I. V., \& Abramowicz, M. A. 1999, MNRAS, 303, 309

Lanzafame, G., Molteni, D., \& Chakrabarti, S. K. 1998, MNRAS, 299, 799

Liang, E. P. T., \& Thompson, K. A. 1980, ApJ, 240, L271

Lu, J. F., Gu, W. M., \& Yuan, F. 1999, ApJ, 523, 340

Mandal, S., \& Chakrabarti, S. K. 2008, ApJ, 689, L17

Molteni, D., Acharya, K., Kuznetsov, O. A., Bisikalo, D., \& Chakrabarti, S. K. 2001, ApJ, 563, L57

Molteni, D., Lanzafame, G., \& Chakrabarti, S. K. 1994, ApJ, 425, 161

Molteni, D., Ryu, D., \& Chakrabarti, S. K. 1996a, ApJ, 470, 460

Molteni, D., Sponholz, H., \& Chakrabarti, S. K. 1996b, ApJ, 457, 805

Molteni, D., Toth, G., \& Kuznetsov, O. A. 1999, ApJ, 516, 411

Nakayama, K. 1992, MNRAS, 259, 259

Narayan, R., \& Yi, I. 1994, ApJ, 428, L13 (NY94)

Nobuta, K., \& Hanawa, T. 1994, PASJ, 46, 257

Paczyński, B., \& Wiita, P. J. 1980, A\&A, 88, 23

Press, W. H., Teukolsky, S. A., Vetterling, W. T., \& Flannery, B. P. 1992,

Numerical Recipes in Fortran (New York: Cambridge Univ. Press)

Ryu, D., Brown, G. L., Ostriker, J. P., \& Loeb, A. 1995, ApJ, 452, 364

Ryu, D., Ostriker, J. P., Kang, H., \& Cen, R. 1993, ApJ, 414, 1

Shakura, N. L., \& Sunyaev, R. A. 1973, A\&A, 24, 337 